\title{
Synthesis and antibacterial activity of new antibiotics arising from cephalosporin-monobactam coupling
}

\author{
Mauro Panunzio, ${ }^{\mathrm{a}}$ Adriano Malabarba, ${ }^{\mathrm{b}}$ and Paola Vicennati ${ }^{\mathrm{a}}$ \\ ${ }^{a}$ CNR-ISOF, Via Gobetti 101, I-40129 Bologna, Italy \\ ${ }^{b}$ Vicuron Pharmaceuticals, Via R. Lepetit, 34, I-21034 Gerenzano, VA, Italy \\ E-mail: mapan@isof.cnr.it
}

\section{This paper is dedicated to Professor Krohn on the occasion of $60^{\text {th }}$ birthday}

(received 13 Apr 04; accepted 18 Aug 04; published on the web 23 Aug 04)

\begin{abstract}
New $\beta$-lactam antibiotics were obtained by coupling the cephalosporin Cefotaxime with monobactams in order to assess the possibility to enhance the cephalosporin activity through a synergistic dual-action mechanism. The activities were tested, in vitro, against a panel of selected bacteria. Preliminary results showed a light change in antibacterial activity when compared with that of the starting cephem counterpart.
\end{abstract}

Keywords: Dual-action, antibiotic, monobactam, cephalosporin

\section{Introduction}

The dual-action mechanism exploited by cephalosporins coupled with other antibiotics has been described. ${ }^{1,2}$ It consists of a primary interaction between the $\beta$-lactam ring of the cephem counterpart with penicillin binding proteins or $\beta$-lactamases, that results in the release of the other antibiotic moiety in position 3 , following a chemical displacement mechanism (Scheme 1).

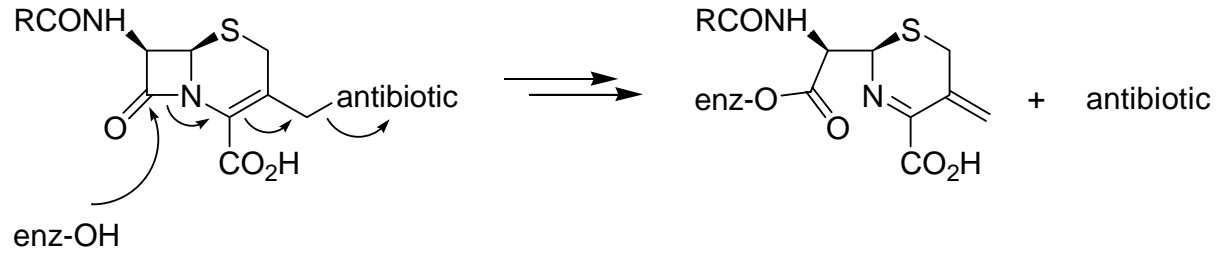

Scheme 1. Dual action mechanism of a generic cephalosporin coupled with another antibiotic. 
So far, with cephem-quinolones ${ }^{3-5}$ (Figure 1), the dual-action mechanism has involved two active moieties inhibiting different molecular targets: i.e. cephalosporin exploiting a cell wall activity, ${ }^{6,7}$ quinolone acting inside the cytoplasmic membrane at the DNA level. ${ }^{8,9}$

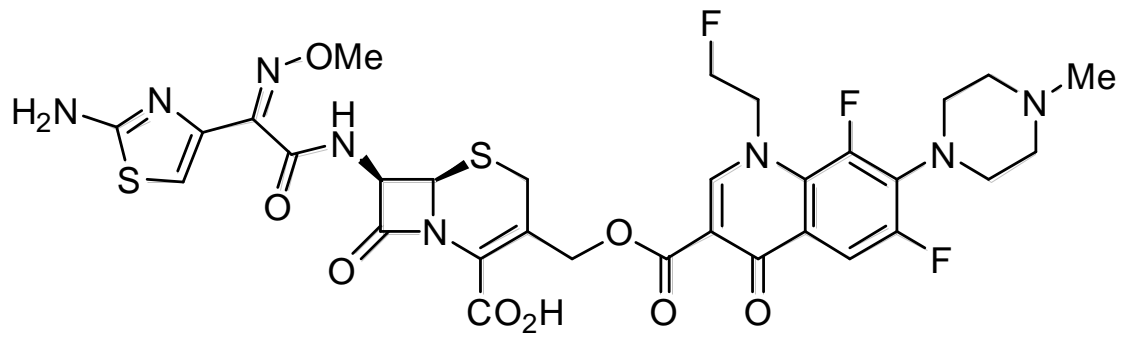

Ro 23-9424

Figure 1. A cephem-quinolone dual action antibiotic.

To the best of our knowledge, no dual action products arising from the coupling between two $\beta$-lactam moieties have been reported, so far. In the present paper we describe the synthesis and the antibacterial activity of new compounds deriving from the above reported chemical linkage. A cephem and a monobactam moiety, both acting against bacterial cell wall targets, were used as parent compounds.

The expected dual action of the cephem-monobactam molecule and the monobactam, when released after enzymatic displacement, was based on the possible synergism resulting from a simultaneous action of these two moieties against different penicillin binding proteins (PBPs). ${ }^{10}$

The chosen $\beta$-lactam moieties have been Cefotaxime ${ }^{11}$ (see Table 2), well known for its antibacterial activity, and monobactams 11, $(E)-12$ and $(Z)-12$. Monobactam $(Z)-7$ is the commercially available antibiotic drug Aztreonam ${ }^{\circledR}, 12$ especially used against Gram-negative aerobic organisms. The structures of unknown monobactams 11, $(E)-\mathbf{1 2}$ and $(Z)-\mathbf{1 2}$ were designed on the basis of the following considerations: (1) The 3-amidic and 4-methyl substituents in trans geometry mimic the structure of Aztreonam ${ }^{\circledR} .^{12}$ (2) The tetrazole ring, successfully used as a $\beta$-lactam ring activating group, ${ }^{13,14}$ was preferred over the sulfonic acid anion, present at the N-1 position of the Aztreonam ${ }^{\circledR}$. This neutral group, in fact, did not introduce further charges in the cephem-monobactam molecule, charges that could make difficult its penetration through the bacterial cell wall. (3) A pyridyl group at the 3-C substituent has been already used in $\mathrm{N}$-(2H-tetrazol-5-yl)-azetidin-2-ones ${ }^{14}$ obtaining monobactam with antibacterial activity. Furthermore, its introduction in the skeleton of monobactam has allowed linking the monobactam to the cephem as 3' quaternary ammonium salts. Compounds of this type have been shown to be a "third generation" antibacterial agents with excellent activity against a wide variety of Gram-positive and Gram-negative pathogens. ${ }^{15}$ 


\section{Results and Discussion}

The synthesis of a series of coupling products $(Z)-\mathbf{1},(Z, E)-\mathbf{2},(Z, Z)-\mathbf{2},(Z, Z)-\mathbf{3}$, derived from Cefotaxime and monobactams, 11, $(E)-\mathbf{1 2}$ and $(Z)-\mathbf{1 2},(Z)-7$, was carried out (see Scheme 4, Table 1). Compound (Z)-4 deriving from Cefotaxime and pyridine was synthesised for comparison. The activity of compounds $(Z)-\mathbf{1},(Z, E)-2,(Z, Z)-2,(Z, Z)-3$ and $(Z)-4$ against a panel of selected bacteria was tested.

The preparation of the unknown $\beta$-lactams 5, (E)-6 and (Z)-6 , employed as 3'-cephalosporin

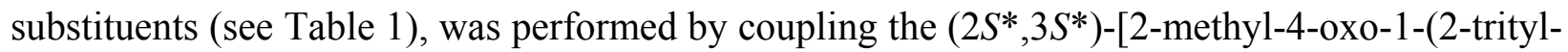
$2 \mathrm{H}$-tetrazol-5-yl)-azetidin-3-yl]carbamic acid tert-butyl ester 8 [obtained from (d,1)-threonine by slight modification of the known procedure $\left.{ }^{14}\right]$ with the appropriate acid (Scheme 2). Deprotection from the trityl protecting group gave the NH-monobactams 11, (E)-12 and (Z)-12.
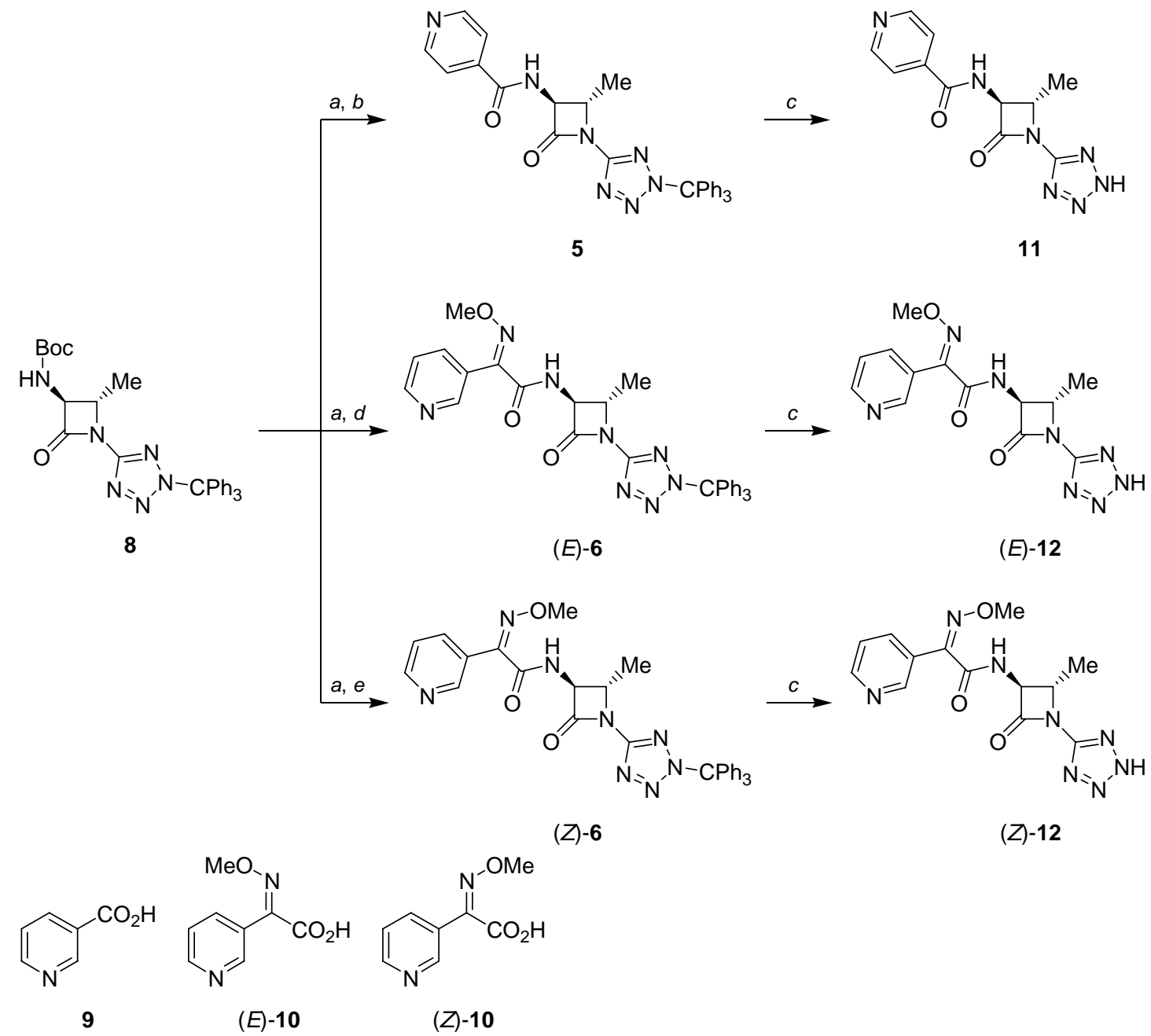

$(Z)-12$

Scheme 2. Reagents and conditions: $a: \mathrm{CF}_{3} \mathrm{CO}_{2} \mathrm{H} ; b$ : MeCN, TEA, EDC,HCl HOBT, 9; c: $\mathrm{HCO}_{2} \mathrm{H}(80 \%)$, acetone; $d$ : MeCN, TEA, EDC,HCl HOBT, (E)-10; $e$ : MeCN, TEA, EDC,HCl HOBT, (Z)-10. 
Table 1. Monobactam moieties and coupling products

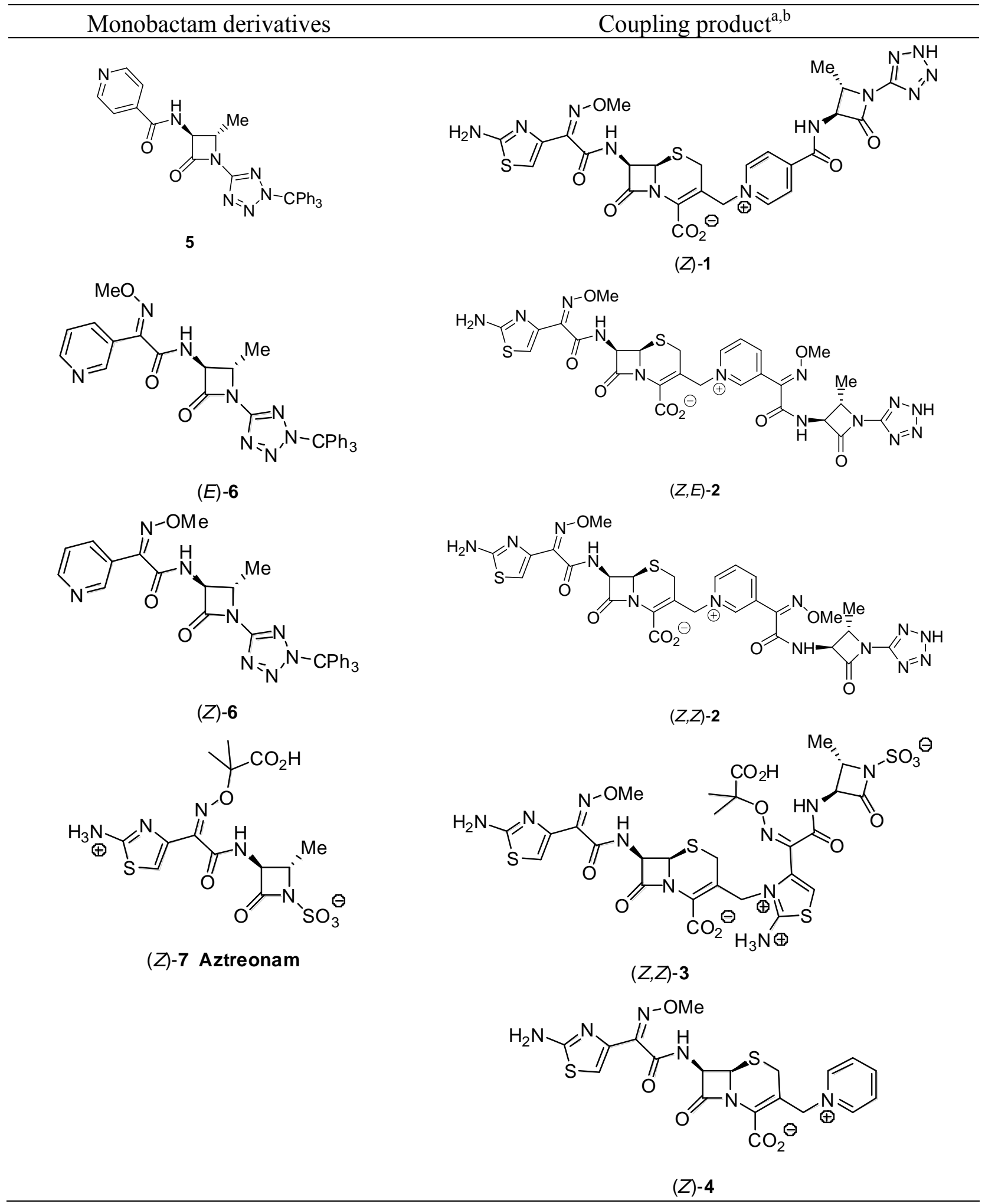

${ }^{a}$ Products 1,2 are diasteromeric mixtures. ${ }^{\mathrm{b}}$ All products 1-4 gave ${ }^{1} \mathrm{H}-\mathrm{NMR}$ and IR spectra consistent with the structure shown. 
The anti and the syn isomers of the methoxyiminopyridin-3-ylacetic acids $(E)-\mathbf{1 0}$ and $(Z)-\mathbf{1 0}$ were obtained by treating oxopyridin-3-ylacetic acid 14 (synthesized by oxidation of 3acetylpyridine $\mathbf{1 3}$ as reported in the literature ${ }^{16}$ ) with $O$-methylhydroxylamine (Scheme 3). After esterification with diazomethane the two isomers were separated by flash chromatography and were subsequently hydrolyzed to the free acids $(E)-\mathbf{1 0}$ and $(Z)-\mathbf{1 0}$ : The configuration was assigned on the basis of the relative rates of methyl ester hydrolysis. In fact, for a series of $\alpha$ alkoxyimino esters it has been demonstrated that the $Z$ isomers (syn) hydrolyze much more slowly than the corresponding $E$ forms (anti). ${ }^{17,18}$

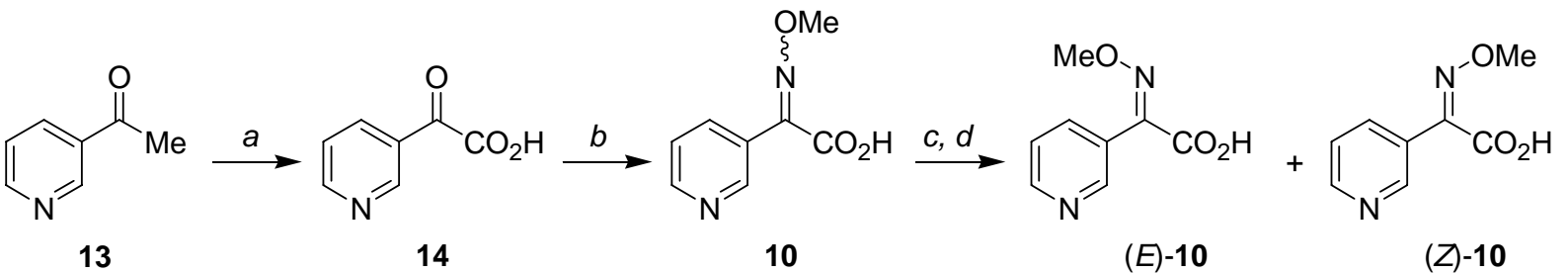

Scheme 3. Reagents and conditions: $a$ : $\mathrm{SeO}_{2}$, pyridine (40\%); b: $\mathrm{MeONH}_{2} \cdot \mathrm{HCl}, \mathrm{NaHCO}_{3}(5 \%$ aqueous solution); $c$ : $\mathrm{CH}_{2} \mathrm{~N}_{2}$, separation of methyl esters by flash chromatography; $d \mathrm{NaOH}$, $\mathrm{MeOH}$.

The coupling products $(Z)-\mathbf{1},(Z, E)-2,(Z, Z)-2,(Z, Z)-3$ and $(Z)-4$ are quaternary cephalosporin derivatives and were prepared by modification of the general method of Bonjouklian and Phillips (in Scheme 4 the synthesis of $(Z, E)-2$ is reported as a typical example). ${ }^{15,19}$ Silylation of Cefotaxime 15 in acetonitrile with $N$-methyl- $N$-(trimethylsilyl)trifluoroacetamide (MSTFA) was followed by in situ formation of the 3 '-iodide derivative with trimethylsilyliodide (TMSI). Excess of trimethylsilyliodide was destroyed by addition of tetrahydrofuran. The 3'-iodide was then replaced by the pyridin-containing monobactam $(E)-6$ in acetonitrile. The silylated cephem was hydrolyzed by treatment with water, and concomitant detritylation of the tetrazole ring on the monobactam moiety occurred.

Cefotaxime-monobactam derivatives (Z)-1, $(Z, E)-2,(Z, Z)-2,(Z, Z)-3$ and (Z)-4 were tested for their in vitro activity in comparison with Cefotaxime, with compound (Z)-7 (Aztreonam) ${ }^{\circledR}$ and with the free monobactam partners 11, $(E)-12$ and $(Z)-12$. Despite the lack of any in vitro activity of monobactams 11, $(E)-12$ and $(Z)-12(M I C>128 \mu \mathrm{g} / \mathrm{mL})$ the coupling compounds $(Z)-1,(Z, E)-$ 2, $(Z, Z)-2$ were tested against some selected bacteria. As a matter of fact, the inactivity in vitro of the monobactam counterpart, against the whole bacterial cell, might have been ascribed to difficulty of penetration through the bacterial cell wall. In contrast, the coupling compounds $(Z)$ 1, $(Z, E)-2,(Z, Z)-2$ could have been able to exploit their potential intrinsic activity on their PBP targets, once entered the bacterial cell wall. Further studies are planned to understand whether the lack of activity of monobactam $(E)-\mathbf{1 2}$ and $(Z)-\mathbf{1 2}$ has to be ascribed to a lack of intrinsic activity or a difficult penetration through the Gram-negative outer membrane. Compounds $(Z)-1$, $(Z, E)-2,(Z, Z)-2,(Z, Z)-3$ showed better activity with respect to compound $(Z)-4$, which is a poor antibacterial agent. However, although maintaining some antibacterial activity, compound (Z)-1 
was significantly less active than Cefotaxime. By contrast, the antimicrobial activity of compounds $(Z, E)-2,(Z, Z)-2$ against most of bacterial strains matched that of Cefotaxime (Table 2 ), and compound $(Z, Z)-2$ was even somewhat more active than Cefotaxime against one strain of $E$. coli and one strain of $K$. pneumoniae. Since compounds $(Z, E)-2,(Z, Z)-2$ differ only in the geometry of the monobactam moiety's methoxy-imine, their chemophysical properties have to be very similar. Thus, if the activity of these compounds had to be ascribed only to the cephem nature without any concomitant dual action, it would be hard to understand the differences between the antibacterial activity of $(Z, E)-2,(Z, Z)-2$. Otherwise, stating the occurrence of the dual action mechanism, these differences can be ascribed to the release of an inactive monobactam moiety in the case of the compound $(Z, E)-2$ and of an active one in the case of the compound $(Z, Z)-2$. Since this dual-action mechanism should depend on the PBP's and/or $\beta$ lactamases, it could happen that it is exploited to a different extent in different bacterial species and strains. In contrast, compound (Z,Z)-3, derived from coupling of Cefotaxime with Aztreonam (Z)-7, was less active than either Cefotaxime or Aztreonam alone. This poor activity could be caused by a difficult cell wall penetration due to the total negative charge. Furthermore, the comparison of its activity with that of Aztreonam, that is better against Gram- positive organisms but worst against Gram-negative, may suggest that compound (Z,Z)-3 maintains a cephalosporin-type spectrum of activity without significantly releasing the monobactam counterpart, as a consequence of a possible preferred primary interaction between the monobactam-ring and its target PBPs in Gram negative bacteria.
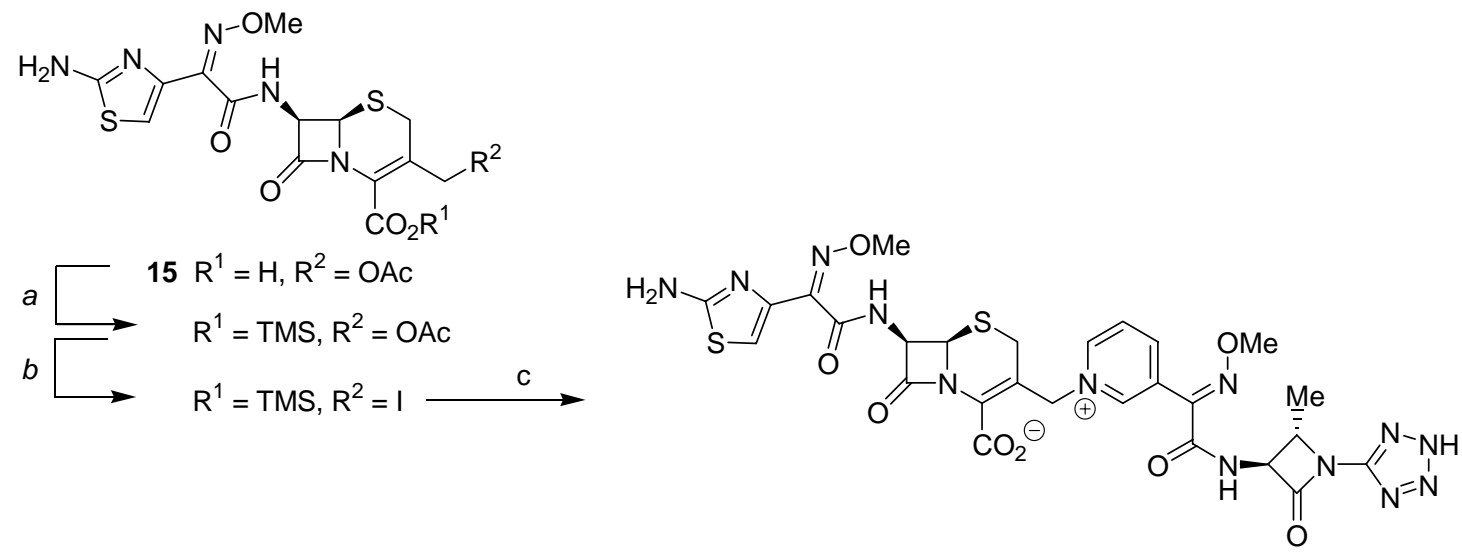

$(Z, E)-2$

Scheme 4. Reagents and conditions: $a$ : MSTFA, MeCN; $b$ : TMSI, THF; $c$ : (E)-6, MeCN followed by $\mathrm{H}_{2} \mathrm{O}$.

These results strongly suggest that the expected dual-action mechanism is at least partially exploited. Work is in progress in order to assess the possibility of exploiting a dual action mechanism by coupling different $\beta$-lactam-antibiotics. 
Table 2. In Vitro antibacterial activity $\left(\mathrm{MIC}^{\mathrm{a}}, \mu \mathrm{g} / \mathrm{mL}\right)$ of the most representative compounds

\begin{tabular}{|c|c|c|c|c|c|c|}
\hline Organism & $\begin{array}{l}\text { Ref. Compd } \\
\text { Cefotaxime }\end{array}$ & $\begin{array}{c}\text { Ref. } \\
\text { Compd. } 4\end{array}$ & $\begin{array}{l}\text { Ref. Compd. } \\
\text { Aztreonam } 7 \\
\end{array}$ & $(Z, E)-2$ & $(Z, Z)-2$ & $(Z, Z)-3$ \\
\hline S.enteriditis ${ }^{\mathrm{b}}$ UAA11RX & 0.125 & 4 & 0.125 & 0.5 & 1 & 1 \\
\hline E.coli ${ }^{\mathrm{b}} \mathrm{K} 12$ & 0.125 & 1 & 0.125 & 0.25 & 0.5 & 1 \\
\hline E.coli ${ }^{\mathrm{b}}$ TEM2 & 0.125 & 16 & 0.5 & 8 & 2 & 4 \\
\hline E coli ${ }^{\mathrm{b}}$ TEM4 & 128 & $>128$ & 32 & $>128$ & 64 & $>128$ \\
\hline E coli $^{\mathrm{b}} \mathrm{Cl}$. Is. & 32 & $>128$ & 128 & 64 & 16 & $>128$ \\
\hline K.pneumoniae $\mathrm{b}^{\mathrm{b}} \mathrm{Cl}$. Is. & 128 & $>128$ & $>128$ & 128 & 8 & $>128$ \\
\hline P.vilgaris ${ }^{\mathrm{b}}$ ATCC 881 & 0.125 & 1 & 0.125 & 1 & 0.25 & 0.25 \\
\hline P.mirabilis ${ }^{\mathrm{b}} \mathrm{Cl}$. Is. & 0.125 & 1 & 0.125 & 0.25 & $<0.125$ & 0.25 \\
\hline P.aeruginosa ${ }^{\mathrm{b}}$ ATCC 10145 & 16 & 32 & 16 & 128 & 128 & 64 \\
\hline E.cloacae $^{\mathrm{b}}$ DER & $>128$ & $>128$ & 128 & n.t. & n.t. & $>128$ \\
\hline $\begin{array}{l}\text { S.aureus } \\
\beta \text {-lactamase producer }\end{array}$ & 16 & 16 & $>128$ & 32 & 16 & 32 \\
\hline S.aureus ${ }^{\mathrm{c}}$ Smith & 8 & 16 & $>128$ & 8 & 8 & 32 \\
\hline S.aureus $^{\mathrm{c}}$ Met-R Cl. Is. & 128 & 128 & $>128$ & $>128$ & $>128$ & $>128$ \\
\hline S.pyrogenes ${ }^{\mathrm{c}} \mathrm{C} 203$ & 0.125 & 0.25 & 2 & $<0.125$ & 0.25 & 0.125 \\
\hline
\end{tabular}

n.t. $=$ not tested.

${ }^{\mathrm{a}} \mathrm{MIC}=$ Minimum inhibitory concentration. ${ }^{\mathrm{b}}$ gram-negatives. ${ }^{\mathrm{c}}$ gram-positives.

\section{Experimental Section}

General Procedures. All starting compounds, unless otherwise stated, were purchased. Reactions were run under an atmosphere of dry nitrogen or argon. FT-IR Spectra were recorded on a Perkin-Elmer infrared spectrometer, mass spectra at $70 \mathrm{eV}$, using the electron impact mode were obtained on Finnigan MAT GCQ instrument, NMR spectra on spectrometers Varian VXR 200, Varian Gemini 300, or Varian Mercury $400 \mathrm{MHz}$ using the residual signal of the solvent as internal standard. HPLC analysis were carried out using a HP 1100 instrument, column Hibar Lichrospher $100 \mathrm{RP}-18(5 \mu \mathrm{m})$, eluting with a gradient from $\mathrm{KH}_{2} \mathrm{PO}_{4}$ buffer $(0.01 \mathrm{~N}, \mathrm{pH} 3.2)$ to $\mathrm{MeCN} / \mathrm{KH}_{2} \mathrm{PO}_{4}$ buffer 85/15. 
(E) and (Z)-2-(Methoxyimino)-2-(3-pyrid-3-yl)acetic acid [(E)-10] and [(Z)-10]. O-Methylhydroxylamine hydrochloride $(8.3 \mathrm{~g}, 98 \mathrm{mmol}, 32.5 \mathrm{~mL}$ of a $25 \%$ solution in water) was added at room temperature and stirring to oxopyridin-3-ylacetic acid $14(3.7 \mathrm{~g}, 24 \mathrm{mmol})$. The $\mathrm{pH} 5 \mathrm{was}$ adjusted by adding a saturated solution of $\mathrm{NaHCO}_{3}$. The resulting mixture was stirred over night affording a homogeneous solution ( $\mathrm{pH} 5.2) .1 \mathrm{~N} \mathrm{HCl}$ was added to adjust $\mathrm{pH} 4$, and the water was removed in vacuo to afford a white solid. This product was dissolved in methanol and heated to $60{ }^{\circ} \mathrm{C}$ under stirring. The precipitate was separated by decantation and was a mixture of (E)-10 and (Z)-10, which was trearted diazomethane. Separation of the corresponding methyl esters by flash chromatography (cyclohexane/ethyl acetate 7:3) followed by hydrolysis with $\mathrm{NaOH} / \mathrm{MeOH}$, and subsequent addition of $\mathrm{HCl}(\mathrm{aq})$ to adjust $\mathrm{pH} 4.2$ ) afforded the acids (E)-10 (2.54 g, 58\%) and (Z)-10 (0.52g, 12\%).

(E)-10. Colorless crystals, mp $115-120{ }^{\circ} \mathrm{C}$. IR (nujol): $1642,1445 \mathrm{~cm}^{-1} ;{ }^{1} \mathrm{H}$ NMR (DMSO-d $d_{6}$, $400 \mathrm{MHz}): \delta 13.40(\mathrm{bs}, 1 \mathrm{H}), 8.56(\mathrm{~m}, 2 \mathrm{H}), 7.81(\mathrm{~m}, 1 \mathrm{H}), 7.45(\mathrm{~m}, 1 \mathrm{H}), 3.87(\mathrm{~s}, 3 \mathrm{H}) .{ }^{13} \mathrm{C}$ NMR (DMSO- $\left.d_{6}, 400 \mathrm{MHz}\right): \delta 164.4,150.5,149.9,147.9,137.4,126.9,123.8,63.9 . \mathrm{MS}: \mathrm{m} / \mathrm{e}(\%): 180$ (10), 149(45), 135, 120(75), 104(100), 77(90). Anal. Calcd. For $\mathrm{C}_{8} \mathrm{H}_{8} \mathrm{~N}_{2} \mathrm{O}_{3}$ (180.05): C, 53.33; H, 4.48; N, 15.55. Found: C, 53.14; H, 4.50; N, 15.47.

(Z)-10. Colorless crystals, mp 125-130 ${ }^{\circ} \mathrm{C}$. IR (nujol): $1643,1598 \mathrm{~cm}^{-1}$; ${ }^{1} \mathrm{H}$ NMR (DMSO- $d_{6}$, $400 \mathrm{MHz}): \delta 8.53(\mathrm{~s}, 1 \mathrm{H}), 8.45(\mathrm{~d}, J=4.6 \mathrm{~Hz}, 1 \mathrm{H}), 7.75(\mathrm{~d}, J=7.4 \mathrm{~Hz}, 1 \mathrm{H}), 7.35(\mathrm{dd}, J=7.4$, $4.6 \mathrm{~Hz}, 1 \mathrm{H}), 3.76(\mathrm{~s}, 3 \mathrm{H}) .{ }^{13} \mathrm{C}$ NMR (DMSO-d, $\left.100 \mathrm{MHz}\right): \delta 165.7,155.1,149.2,137.0,129.7$, 123.4, 123.3, 62.2. MS: m/e (\%): 180 (10), 149(45), 135, 120(75), 104(100), 77(90). Anal. Calcd. For $\mathrm{C}_{8} \mathrm{H}_{8} \mathrm{~N}_{2} \mathrm{O}_{3}$ (180.05): C, 53.33; H, 4.48; N, 15.55. Found: C, 53.34; H, 4.55; N, 15.42.

\section{(Z)-N1-[(2R*,3R*)-2-Methyl-4-oxo-1-(2-trityl-2H-1,2,3,4-tetrazol-5-yl)azetidin-3-yl]-2-} (methoxyimino) -2-(3-pyridyl)acetamide $[(Z)-6]$. Typical procedure for the preparation of $\beta$-lactams 5, 6

A cooled $\left(0^{\circ} \mathrm{C}\right)$ solution of $N$-[(2S*,3 $\left.S^{*}\right)$-2-methyl-4-oxo-1-(2-trityl-2H-tetrazol-5-yl)azetidin-3yl]carbamic acid tert-butyl ester $8(227 \mathrm{mg}, 0.45 \mathrm{mmol})$ in trifluoroacetic acid $(99 \%, 3 \mathrm{~mL})$ was allowed to warm to room temperature within $10 \mathrm{~min}$ under a stream of nitrogen. The trifluoroacetic acid was then removed in vacuo at room temperature affording a yellow oil. This product was dissolved under nitrogen in dry acetonitrile $(15 \mathrm{~mL})$, and, at $0{ }^{\circ} \mathrm{C}$ triethylamine (1.575 mL, $1.13 \mathrm{mmol}), 1$-(3-dimethylaminopropyl)-3-ethylcarbodiimide hydrochloride (94 mg, $0.5 \mathrm{mmol}), 1$-hydroxybenzotriazole $(78 \mathrm{mg}, 0.59 \mathrm{mmol})$ and $(Z)-2-($ methoxyimino)-2-(3-pyrid-3yl)acetic acid (200 mg, 0,39 mmol) (Z)-10 were added. The reaction mixture was stirred at room temperature overnight, was then poured into an aqueous solution of sodium carbonate $(5 \mathrm{~mL}, 5 \%$ solution) and extracted with dichloromethane $(3 \times 15 \mathrm{~mL})$. The organic layer was dried over anhydrous sodium sulfate and evaporated under vacuo. (Z)-6 was isolated as pure product by flash chromatography (cyclohexane/ethyl acetate 3/7).

Colorless crystals $(24 \%)$, mp $145-148{ }^{\circ} \mathrm{C}$. IR $\left(\mathrm{CDCl}_{3}\right): 3377,1770 \mathrm{~cm}^{-1} ;{ }^{1} \mathrm{H}$ NMR $\left(\mathrm{CDCl}_{3}\right.$, $400 \mathrm{MHz}$ ): $\delta 8.83$ (bs, 1H), $8.53(\mathrm{~m}, 1 \mathrm{H}), 7.95(\mathrm{~m}, 1 \mathrm{H}), 7.44(\mathrm{~m}, 1 \mathrm{H}), 7.40-7.21(\mathrm{~m}, 11 \mathrm{H}), 7.12$ $(\mathrm{m}, 5 \mathrm{H}), 4.83\left(\mathrm{dd}, J_{1}=6.4 \mathrm{~Hz}, J_{2}=2.8 \mathrm{~Hz}, 1 \mathrm{H}\right), 4.36(\mathrm{~m}, 1 \mathrm{H}), 4.11(\mathrm{~s}, 3 \mathrm{H}), 1.70(\mathrm{~d}, J=6.2 \mathrm{~Hz}$, $3 \mathrm{H}) .{ }^{13} \mathrm{C} \mathrm{NMR}\left(\mathrm{CDCl}_{3}, 100 \mathrm{MHz}\right): \delta 161.6,161.1,156.7,149.7,148.3,146.8,140.7,130.2$, 
128.4, 127.9, 127.8, 127.7, 127.1, 83.7, 63.6, 58.2, 21.6, 17.0. Anal. Calcd for $\mathrm{C}_{32} \mathrm{H}_{28} \mathrm{~N}_{8} \mathrm{O}_{3}$ (572.62): C, 67.12; H, 4.93; N, 19.5. Found: C, 67.29; H, 4.96; N, 19.40.

Following the same procedure and using the appropriate reagents products (5) and (E)-6 were obtained in the yields reported in square brackets.

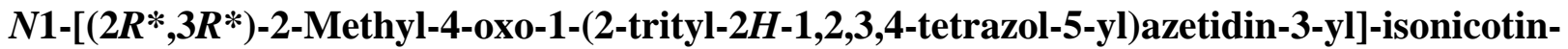
amide (5). Pale yellow crystals (25\%), mp $170{ }^{\circ} \mathrm{C}$ (dec.). IR (film): $1786 \mathrm{~cm}^{-1} ;{ }^{1} \mathrm{H} \mathrm{NMR}\left(\mathrm{CDCl}_{3}\right.$, $200 \mathrm{MHz}): \delta 8.71(\mathrm{~d}, J=5.9 \mathrm{~Hz}, 2 \mathrm{H}), 8.07(\mathrm{~d}, J=7.0 \mathrm{~Hz}, 1 \mathrm{H}), 7.75(\mathrm{~d}, J=5.9 \mathrm{~Hz}, 2 \mathrm{H}), 7.41-$ $7.18(\mathrm{~m}, 10 \mathrm{H}), 7.04(\mathrm{~m}, 5 \mathrm{H}), 4.96\left(\mathrm{dd}, J_{1}=7.0 \mathrm{~Hz}, J_{2}=2.4 \mathrm{~Hz}, 1 \mathrm{H}\right), 3.94(\mathrm{~m}, 1 \mathrm{H}), 1.59(\mathrm{~d}, J=$ $6.2 \mathrm{~Hz}, 3 \mathrm{H}) .{ }^{13} \mathrm{C} \mathrm{NMR}\left(\mathrm{CDCl}_{3}, 100 \mathrm{MHz}\right): \delta 165.5,162.6,156.5,150.5,140.6 .139 .8,130.2$, 128.5, 127.8, 121.2, 83.9, 64.0, 59.1, 17.1. MS: m/z (\%): 514, 459 (10), 243 (100), 165 (50), 106, 78. Anal. Calcd for $\mathrm{C}_{30} \mathrm{H}_{25} \mathrm{~N}_{7} \mathrm{O}_{2}$ (515.57): C, 69.89; H, 4.89; N, 19.02 Found: C, 70.09; H, 4.91; $\mathrm{N}, 19.00$.

(E)-N1-[(2R*,3R*)-2-Methyl-4-oxo-1-(2-trityl-2H-1,2,3,4-tetrazol-5-yl)azetidin-3-yl]-2(methoxyimino)-2-(3-pyridyl)acetamide [(E)-6]. Pale yellow crystals $(22 \%), \mathrm{mp} 185^{\circ} \mathrm{C}$ (dec.): IR $\left(\mathrm{CDCl}_{3}\right): 3377,1770 \mathrm{~cm}^{-1} ;{ }^{1} \mathrm{H}$ NMR $\left(\mathrm{CDCl}_{3}, 200 \mathrm{MHz}\right): \delta 8.67$ (bs, $\left.1 \mathrm{H}\right), 8.62(\mathrm{bs}, 1 \mathrm{H}), 7.80$ $(\mathrm{m}, 1 \mathrm{H}), 7.63(\mathrm{~m}, 1 \mathrm{H}), 7.35(\mathrm{~m}, 11 \mathrm{H}), 7.12(\mathrm{~m}, 5 \mathrm{H}), 4.81\left(\mathrm{dd}, J_{1}=7.04 \mathrm{~Hz}, J_{2}=3.52 \mathrm{~Hz}, 1 \mathrm{H}\right)$, $4.33(\mathrm{~m}, 1 \mathrm{H}), 4.01(\mathrm{~s}, 3 \mathrm{H}), 1.66(\mathrm{~d}, J=6.16 \mathrm{~Hz}, 3 \mathrm{H}) .{ }^{13} \mathrm{C} \mathrm{NMR}\left(\mathrm{CDCl}_{3}, 100 \mathrm{MHz}\right): \delta 162.3$, $161.6,157.3,150.1,146.2,141.2,140.8,137.6,130.9$, 130.3, 128.4, 128.3, 127.8, 124.3, 60.4, 58.2, 29.2, 17.3. Anal. Calcd for $\mathrm{C}_{32} \mathrm{H}_{28} \mathrm{~N}_{8} \mathrm{O}_{3}$ (572.62): C, 67.12; H, 4.93; N, 19.5. Found: C, $67.39 ; \mathrm{H}, 4.95 ; \mathrm{N}, 19.49$.

N1-[(2R*,3R*)-2-Methyl-4-oxo-1-(-2H-1,2,3,4-tetrazol-5-yl)azetidin-3-yl]-isonicotinamide (11): Typical procedure for the preparation of $\beta$-lactams 11, 12. To a suspension of 5 (60 mg, $0.12 \mathrm{mmol})$ in acetone $(10 \mathrm{~mL})$ was added formic acid $(80 \%, 1 \mathrm{~mL}$ each) in two portions. After $6 \mathrm{~h}$ the solution was dried in vacuo, and the resulting solid was washed with diethyl ether to eliminate triphenylmethanol. 12 was obtained as white solid $(31 \mathrm{mg}, 95 \%), \mathrm{mp} 190{ }^{\circ} \mathrm{C}$ (dec.). IR (nujol): 2923, 2853, 1766, $1680 \mathrm{~cm}^{-1} ;{ }^{1} \mathrm{H}$ NMR (DMSO-d $6,200 \mathrm{MHz}$ ): $\delta 9.52$ (d, $J=7.4 \mathrm{~Hz}$, $1 \mathrm{H}), 8.69(\mathrm{~d}, J=5.9 \mathrm{~Hz}, 2 \mathrm{H}), 7.69(\mathrm{~d}, J=5.9,2 \mathrm{H}), 4.85\left(\mathrm{dd}, J_{1}=2.9 \mathrm{~Hz}, J_{2}=7.4 \mathrm{~Hz}, 1 \mathrm{H}\right), 4.37$ (m, 1H), 3.28 (bs, $1 \mathrm{H}), 1.53$ (d, $J=6.2 \mathrm{~Hz}, 3 \mathrm{H}) .{ }^{13} \mathrm{C}$ NMR (DMSO-d, $\left.100 \mathrm{MHz}\right): \delta 164.9,163.1$, 156.0, 150.6, 139.8, 121.2, 63.8, 56.5, 16.3. Mz (m/z): 258(30) $\left(\mathrm{M}^{+}-15\right), 243(35), 229,205$, 162(65), 147(100), 106, 78. Anal. Calcd for $\mathrm{C}_{11} \mathrm{H}_{11} \mathrm{~N}_{7} \mathrm{O}_{2}$ (273.25): C, 48.35; H, 4.06; N, 35.88. Found: C, 48.51; H, 4.08; N, 35.75.

Following the same procedure and starting from $(E)-\mathbf{6}$ and $(Z)-\mathbf{6}$ products $(E)-\mathbf{1 2}$ and $(Z)-12$, respectively, were obtained.

(E)-N1-[(2R*,3R*)-2-Methyl-4-oxo-1-(2H-1,2,3,4-tetrazol-5-yl)azetidin-3-yl]-2-(methoxyimino)-2-(3-pyridyl)acetamide [(E)-12]. Pale yellow crystals $(85 \%), \mathrm{mp} 172-175{ }^{\circ} \mathrm{C}$ (dec.): IR: 1776 (nujol) $\mathrm{cm}^{-1}$; ${ }^{1} \mathrm{H}$ NMR (DMSO-d $\left.{ }_{6}, 400 \mathrm{MHz}\right): \delta 8.78(\mathrm{~d}, J=8.2 \mathrm{~Hz}, 1 \mathrm{H}), 8.71(\mathrm{~d}, J=$ $1.2 \mathrm{~Hz}, 1 \mathrm{H}), 8.62\left(\mathrm{dd}, J_{1}=5.2 \mathrm{~Hz}, J_{2}=1.6 \mathrm{~Hz}, 1 \mathrm{H}\right), 7.91\left(\mathrm{dt}, J_{1}=8.4 \mathrm{~Hz}, J_{2}=1.6 \mathrm{~Hz}, 1 \mathrm{H}\right), 7.47$ $(\mathrm{m}, 1 \mathrm{H}), 5.02\left(\mathrm{dd}, J_{1}=8.20 \mathrm{~Hz}, J_{2}=3.2 \mathrm{~Hz}, 1 \mathrm{H}\right), 4.54(\mathrm{~m}, 1 \mathrm{H}), 4.15(\mathrm{~s}, 3 \mathrm{H}), 1.74(\mathrm{~d}, J=6.4 \mathrm{~Hz}$, 3H). Anal. Calcd for $\mathrm{C}_{13} \mathrm{H}_{14} \mathrm{~N}_{8} \mathrm{O}_{3}$ (330.12): C, 47.27; H, 4.27; N, 32.92. Found: C, 47.47; H, $4.30 ; \mathrm{N}, 32.75$. 
(Z)-N1-[(2R*,3R*)-2-Methyl-4-oxo-1-(2H-1,2,3,4-tetrazol-5-yl)azetidin-3-yl]-2-(methoxyimino)-2-(3-pyridyl)acetamide [(Z)-12]. Pale yellow crystals $(90 \%), \mathrm{mp} 183-186{ }^{\circ} \mathrm{C}$ (dec.): ${ }^{1} \mathrm{H}$ NMR (DMSO-d $\left.{ }_{6}, 400 \mathrm{MHz}\right): \oint 8.80(\mathrm{~s}, 1 \mathrm{H}), 8.79(\mathrm{bs}, 1 \mathrm{H}), 8.63(\mathrm{~m}, 1 \mathrm{H}), 8.01$ (dt, $J_{1}=8.2 \mathrm{~Hz}$, $\left.\mathrm{J}_{2}=2.0 \mathrm{~Hz}, 1 \mathrm{H}\right), 7.45,\left(\mathrm{dd}, J_{1}=8.2 \mathrm{~Hz}, J_{2}=4.8 \mathrm{~Hz}, 1 \mathrm{H}\right), 4.99(\mathrm{~m}, 1 \mathrm{H}), 4.64(\mathrm{~m}, 1 \mathrm{H}), 4.01(\mathrm{~s}$, $3 \mathrm{H}), 1.75(\mathrm{~d}, J=6.0 \mathrm{~Hz}, 3 \mathrm{H})$. Anal. Calcd for $\mathrm{C}_{13} \mathrm{H}_{14} \mathrm{~N}_{8} \mathrm{O}_{3}$ (330.12): C, 47.27; H, 4.27; N, 32.92 . Found: C, 47.50; H, 4.38; N, 32.75.

(Z)-(7R,7aR)-7-[2-(2-Amino-1,3-thiazol-4-yl)-2-(methoxyimino)acetyl]amino-3-[3-[(Z)-N1[(2R*,3S*)-2-methyl-4-oxo-1-(2H-1,2,3,4-tetraazol-5-yl)-azetidin-3-yl]-2-(methoxyimino)acetamide-2-yl]-1-pyridiniumyl]methyl-6-oxo-7,7a-dihydro-2H,6H-azeto[2,1-b][1,3]thiazine-4-carboxylate $[(Z, Z)-2]$. Typical procedure for the preparation of quaternary cephalosporins 1-4. Ceftaxime 15 (45 mg, $0.1 \mathrm{mmol})$ was suspended in acetonitrile (4 mL) under a nitrogen atmosphere at $5-10{ }^{\circ} \mathrm{C} . \mathrm{N}$-Methyl- $N$-(trimethylsilyl)trifluoroacetamide $(49 \mu \mathrm{L}$, $0.35 \mathrm{mmol}$ ) was added and the mixture was stirred at $5-15{ }^{\circ} \mathrm{C}$ for $2 \mathrm{~h}$. To the resulting homogeneous solution of cephem trimethylsilyl ester was added trimethylsilyliodide ( $49 \mu \mathrm{L}$, $0.35 \mathrm{mmol}$ ). The solution was stirred at $10{ }^{\circ} \mathrm{C}$ for $35 \mathrm{~min}$ and was then allowed to warm to room temperature. Excess of TMSI and the solvent were evaporated in vacuo to afford 3'iodomethylcephem as viscous oil. This oil was dissolved in acetonitrile $(4 \mathrm{~mL})$, tetrahydrofuran $(37 \mu \mathrm{L}, 0.42 \mathrm{mmol})$ was added to destroy the excess of TMSI, and the solution was stirred at room temperature for $10 \mathrm{~min}$. To the solution $(Z)-6(63 \mathrm{mg}, 0.1 \mathrm{mmol})$ was added in one portion. The reaction was stirred at room temperature for $3 \mathrm{~h}$ until a precipitate separated. Water $(4 \mu \mathrm{L}$, $0.24 \mathrm{mmol}$ ) was added and the precipitate was separated from the solution by decantation. Trituration with diethyl ether and acetone and drying in vacuo afforded product (Z,Z)-2 (18.5 $\mathrm{mg}, 25 \%$ ) of sufficient spectroscopic purity for biological tests. Due to the difficulty of separation of diastereomeric mixtures the products $(Z)-\mathbf{1},(Z, E)-\mathbf{2}$ and $(Z, Z)-\mathbf{2}$ were tested as such. Spectroscopic data, including the ${ }^{1} \mathrm{H}$ NMR are given below. The signals of the minor isomer are given in square brackets.

(Z,Z)-2. Pale yellow crystals, mp $197-200^{\circ} \mathrm{C}$ (dec.). IR (nujol) $1765 \mathrm{~cm}^{-1} .{ }^{1} \mathrm{H}$ NMR (DMSO- $d_{6}$, $400 \mathrm{MHz}$ ): $\delta 9.62$ (d, $J=7.6 \mathrm{~Hz}, 1 \mathrm{H}), 9.58$ (d, $J=7.2 \mathrm{~Hz}, 1 \mathrm{H}), 8.72$ (bs, 1H), 8.68 (bs, 1H), 7.96 $(\mathrm{m}, 1 \mathrm{H}), 7.55(\mathrm{~m}, 1 \mathrm{H}), 7.20(\mathrm{bs}, 2 \mathrm{H}), 6.74(\mathrm{~s}, 1 \mathrm{H}),[6.73(\mathrm{~s})],\left[5.78\left(\mathrm{dd}, J_{1}=8.4 \mathrm{~Hz}, J_{2}=\right.\right.$ $4.8 \mathrm{~Hz})],[5.14(\mathrm{~d}, J=4.8 \mathrm{~Hz})], 5.09(\mathrm{~d}, J=4.4 \mathrm{~Hz}, 1 \mathrm{H}), 4.98(\mathrm{~d}, J=13.0 \mathrm{~Hz}, 1 \mathrm{H}),\left[4.87\left(\mathrm{dd}, J_{1}=\right.\right.$ $\left.\left.6.4 \mathrm{~Hz}, J_{2}=3.6 \mathrm{~Hz}\right)\right], 4.83\left(\mathrm{dd}, J_{1}=7.2 \mathrm{~Hz}, J_{2}=4.4 \mathrm{~Hz}, 1 \mathrm{H}\right), 4.67(\mathrm{~d}, J=13.0 \mathrm{~Hz}, 1 \mathrm{H}),[4.44$ (m)], $4.37(\mathrm{~m}, 1 \mathrm{H}), 4.05$ (s, 3H), $3.98(\mathrm{~s}, 3 \mathrm{H}), 3.52$ (d, J=18.0 Hz, 1H), 3.39 (d, J = 18.0 Hz, $1 \mathrm{H}) ;[1.61(\mathrm{~d}, J=6.0 \mathrm{~Hz})], 1.53(\mathrm{~d}, J=6.4 \mathrm{~Hz}, 3 \mathrm{H})$. Anal. Calcd for $\mathrm{C}_{28} \mathrm{H}_{30} \mathrm{~N}_{13} \mathrm{O}_{8} \mathrm{~S}_{2}$ (740.75): C, 45.40; H, 4.08; N, 24.58. Found: C, 45.60; H, 4.09; N, 24.50.

The same procedure employing azetidinones $(E)-6$ and $(Z)-7$ were used for the synthesis of coupling products $\mathbf{1}$ and $\mathbf{2}$. The relative yields are reported in square brackets. 
(Z)-(7R,7aR)-7-[2-(2-Amino-1,3-thiazol-4-yl)-2-(methoxyimino)acetyl]amino-3-[4-[[(2R*, 3S*)-2-methyl-4-oxo-1-(2H-1,2,3,4-tetraazol-5-yl)-3-azetidinyl]aminocarbonyl]-1-pyridiniumyl]methyl-6-oxo-7,7a-dihydro- $2 \mathrm{H}, 6 \mathrm{H}$-azeto[2,1-b][1,3]thiazine-4-carboxylate $\quad[(\mathrm{Z})-1]$. Pale yellow crystals $\left(15 \%\right.$ ), mp $167-172^{\circ} \mathrm{C}$ (dec.). IR (nujol) $1778 \mathrm{~cm}^{-1} .{ }^{1} \mathrm{H}$ NMR (DMSO- $d_{6}$, $400 \mathrm{MHz}): \delta 10.01(\mathrm{~d}, J=7.8 \mathrm{~Hz}, 1 \mathrm{H}), 9.66(\mathrm{~d}, J=7.6 \mathrm{~Hz}, 1 \mathrm{H}), 9.21(\mathrm{~d}, J=6.6 \mathrm{~Hz}, 1 \mathrm{H}), 8.81$ (d, $J=6.0 \mathrm{~Hz}, 1 \mathrm{H}), 8.48$ (d, $J=6.6 \mathrm{~Hz}, 1 \mathrm{H}), 7.84$ (d, $J=6.0 \mathrm{~Hz}, 1 \mathrm{H}), 7.10$ (bs, 2H), [6.83], 6.76 $(\mathrm{s}, 1 \mathrm{H}),[5.90(\mathrm{~m})], 5.86\left(\mathrm{dd}, J_{1}=5.0 \mathrm{~Hz}, J_{2}=7.6 \mathrm{~Hz}, 1 \mathrm{H}\right), 5.66(\mathrm{~d}, J=15.0 \mathrm{~Hz}, 1 \mathrm{H}), 5.56(\mathrm{~d}, J=$ $15.0,1 \mathrm{H}), 5.16(\mathrm{~d}, J=15.0 \mathrm{~Hz}, 1 \mathrm{H}),[5.00(\mathrm{~d}, J=6.8 \mathrm{~Hz})], 4.93\left(\mathrm{dd}, J_{1}=2.8 \mathrm{~Hz}, J_{2}=7.8 \mathrm{~Hz}\right.$, $1 \mathrm{H}),[4.49(\mathrm{~m})], 4.48(\mathrm{~m}, 1 \mathrm{H}),[3.84(\mathrm{~s})], 3.82(\mathrm{~s}, 3 \mathrm{H}), 3.55(\mathrm{~d}, J=16.0 \mathrm{~Hz}, 1 \mathrm{H}), 3.38(\mathrm{~d}, J=$ $16.0 \mathrm{~Hz}, 1 \mathrm{H}),[1.62(\mathrm{~d}, J=6.0)], 1.61(\mathrm{~d}, J=6.0 \mathrm{~Hz}, 3 \mathrm{H})$.

(Z)-(7R,7aR)-7-[2-(2-Amino-1,3-thiazol-4-yl)-2-(methoxyimino)acetyl]amino-3-[3-[(E)-N1[(2R*,3S*)-2-methyl-4-oxo-1-(2H-1,2,3,4-tetrazol-5-yl)-azetidin-3-yl]-2-(methoxyimino)acetamide-2-yl]-1-pyridiniumyl]methyl-6-oxo-7,7a-dihydro-2 $\mathrm{H}, 6 \mathrm{H}$-azeto[2,1-

b][1,3]thiazine-4-carboxylate [(Z,E)-2]. Pale yellow crystals $(23 \%)$, mp $197-200^{\circ} \mathrm{C}$ (dec.). IR (nujol) $1775 \mathrm{~cm}^{-1} .{ }^{1} \mathrm{H}$ NMR (DMSO- $\left.d_{6}, 400 \mathrm{MHz}\right): \delta 9.59(\mathrm{~d}, J=8.0 \mathrm{~Hz}, 1 \mathrm{H}) ; 9.39(\mathrm{~d}, J=$ $8.8 \mathrm{~Hz}, 1 \mathrm{H}), 9.28(\mathrm{bs}, 1 \mathrm{H}), 9.05(\mathrm{~d}, J=4.8 \mathrm{~Hz}, 1 \mathrm{H}), 8.72(\mathrm{~d}, J=8.4 \mathrm{~Hz}, 1 \mathrm{H}), 8.27(\mathrm{~m}, 1 \mathrm{H}), 7.26$ (bs, 2H), [6.73 (s)], $6.70(\mathrm{~s}, 1 \mathrm{H}),\left[5.90\left(\mathrm{dd}, J_{1}=8.4, J_{2}=5.2 \mathrm{~Hz}\right)\right], 5.87\left(\mathrm{dd}, J_{1}=8.0, J_{2}=5.0 \mathrm{~Hz}\right.$, $1 \mathrm{H}), 5.62(\mathrm{~d}, J=14.8,1 \mathrm{H}), 5.54(\mathrm{~d}, J=14.8,1 \mathrm{H}), 5.18(\mathrm{~d}, J=5.0 \mathrm{~Hz}),[5.14(\mathrm{~d}, J=4.8)], 4.96$ $(\mathrm{m}, 1 \mathrm{H}),[4.37, \mathrm{~m}], 4.42(\mathrm{~m}, 1 \mathrm{H}), 4.06(\mathrm{~s}, 3 \mathrm{H})[4.05(\mathrm{~s})],[3.80(\mathrm{~s})], 3.79(\mathrm{~s}, 3 \mathrm{H}), 3.52(\mathrm{~d}, J=$ $18.4 \mathrm{~Hz}, 1 \mathrm{H}), 3.39$ (d, $J=18.4 \mathrm{~Hz}, 1 \mathrm{H}) ; 1.58(\mathrm{~d}, J=7.6 \mathrm{~Hz}, 3 \mathrm{H}),[1.52(\mathrm{~d}, J=5.6 \mathrm{~Hz})]$. Anal. Calcd for $\mathrm{C}_{28} \mathrm{H}_{30} \mathrm{~N}_{13} \mathrm{O}_{8} \mathrm{~S}_{2}$ (740.75): C, 45.40; H, 4.08; N, 24.58. Found: C, 45.65; H, 4.10; N, 24.50.

(Z)-(7R,7aR)-7-[2-(2-Amino-1,3-thiazol-4-yl)-2-(methoxyimino)acetyl]amino-3-[3-[(2-amino)[(Z)(2S,3S)-2-methyl-4-oxo-1-sulfo-azetidin-3-yl]-2-(1-carboxy-1-methylethoxy)iminoacetamide-2-yl]-1,3thiazolinium-2-amino-3-yl]methyl-6-oxo-7,7a-dihydro-2H,6H-azeto[2,1-b][1,3]thiazine-4-carboxylate $[(Z, Z)-3]$. Aztreonam 7 was used as monobactam counterpart following the protocol above reported except for the addition of 1.1 eqiv of diisopropylethylamine for releasing Aztreonam 7 from its zwitterion. Pale yellow crystals (Z,Z)-3 (85\%), mp $208^{\circ} \mathrm{C}$ (dec.). IR (nujol): $1770 \mathrm{~cm}^{-1} .{ }^{1} \mathrm{H}$ NMR (DMSO- $\left.d_{6}, 400 \mathrm{MHz}\right): \delta 9.58(\mathrm{~m}, 2 \mathrm{H}), 7.21(\mathrm{~s}, 1 \mathrm{H}), 7.20(\mathrm{~m}, 4 \mathrm{H}), 6.69(\mathrm{~s}, 1 \mathrm{H}), 6.67\left(\mathrm{dd}, J_{1}=\right.$ $\left.7.6 \mathrm{~Hz}, J_{2}=4.4 \mathrm{~Hz}, 1 \mathrm{H}\right), 5.50(\mathrm{~m}, 1 \mathrm{H}), 5.00(\mathrm{~m}, 2 \mathrm{H}), 4.46\left(\mathrm{dd}, J_{1}=7.6 \mathrm{~Hz}, J_{2}=2.4 \mathrm{~Hz}, 1 \mathrm{H}\right)$, $3.79(\mathrm{~s}, 3 \mathrm{H}), 3.67(\mathrm{~m}, 1 \mathrm{H}), 3.21(\mathrm{~m}, 2 \mathrm{H}), 1.40(\mathrm{~m}, 9 \mathrm{H})$. Anal. Calcd fo $\mathrm{C}_{27} \mathrm{H}_{30} \mathrm{~N}_{10} \mathrm{O}_{13} \mathrm{~S}_{4}(830.09)$ : C, 39.03; H, 3.64; N, 16.86. Found: C, 39.30; H, 4.36; N, 17.00.

(Z)-(7R,7aR)-7-[2-(2-Amino-1,3-thiazol-4-yl)-2-(methoxyimino)acetyl]amino-3-[1-pyridiniumyl]ethyl-6-oxo-7,7a-dihydro-2H,6H-azeto[2,1-b][1,3]thiazine-4-carboxylate $\quad[(Z)-4]$. Following the procedure reported for product 2 and using pyridine instead of monobactam, product (Z)-4 was obtained as yellow crystals (84\%), mp $173{ }^{\circ} \mathrm{C}$ (dec.) IR (nujol) $1774 \mathrm{~cm}^{-1} .{ }^{1} \mathrm{H}$ NMR (DMSO- $\left.d_{6}, 200 \mathrm{MHz}\right): \delta 9.60(\mathrm{~d}, J=8.0 \mathrm{~Hz}, 1 \mathrm{H}), 9.02(\mathrm{~d}, J=5.4 \mathrm{~Hz}, 2 \mathrm{H}), 8.65(\mathrm{~m}, 1 \mathrm{H})$, $8.20(\mathrm{dd}, \mathrm{m}, 2 \mathrm{H}), 7.23(\mathrm{bs}, 2 \mathrm{H}), 6.70(\mathrm{~s}, 1 \mathrm{H}), 5.87\left(\mathrm{dd}, J_{1}=8.0 \mathrm{~Hz}, J_{2}=4.9 \mathrm{~Hz}, 1 \mathrm{H}\right), 5.60(\mathrm{~d}, J=$ $14.5 \mathrm{~Hz}, 1 \mathrm{H}), 5.47(\mathrm{~d}, J=14.5 \mathrm{~Hz}, 1 \mathrm{H}), 5.19(\mathrm{~d}, J=4.9 \mathrm{~Hz}, 1 \mathrm{H}), 3.82(\mathrm{~s}, 3 \mathrm{H}), 3.55$ (d, $J=$ $18.3 \mathrm{~Hz}, 1 \mathrm{H}), 3.35$ (d, $J=1.3 \mathrm{~Hz}, 1 \mathrm{H}) .{ }^{13} \mathrm{C}$ NMR (DMSO-d, $\left.200 \mathrm{MHz}\right): \delta 168.4,163.9,162.9$, 162.8, 148.8, 147.0, 143.3, 145.0, 142.1, 129.3, 128.4, 119.8, 108.9, 61.9, 60.6, 59.0, 57.6. Anal. 
Calcd for $\mathrm{C}_{20} \mathrm{H}_{21} \mathrm{~N}_{6} \mathrm{O}_{5} \mathrm{~S}_{2}$ (489.55): C, 49.07; H, 4.32; N, 17.17. Found: C, 49.30; H, 4.34; N, 17.09 .

\section{References}

1. Hamilton-Miller, J. M. T. J. Antimicrob.Chemother. 1994, 33, 197.

2. Keith, D. D. In Recent Advances in Chemistry of Anti-infective Agents; Royal Society of Chemistry, 1993.

3. Albrecht, H. A.; Beskid, G.; Chan, K. K.; Christensen, J. G.; Cleeland, R.; Deitcher, K. H.; Georgopapadakou, N. H.; Keith, D. D.; D.L., P.; Sepinwall, J.; Specian, A. C., Jr.; Then, R. L.; Weigele, M.; West, K. F.; Yang, R. J. Med. Chem. 1990, 33, 77.

4. Albrecht, H. A.; Beskid, G.; Christensen, J. G.; Durkin, J. W.; Fallat, V.; Georgopapadakou, N. H.; Keith, D. D.; Konzelmann, F. M.; Lipschitz, E. R.; McGarry, D. H.; Siebelist, J.; Wei, C. C.; Weigele, M.; Yang, R. J. Med. Chem. 1991, 34, 669.

5. Okabe, M.; Sun, R. C. Synthesis 1992, 11, 1160.

6. Morin, R. B.; Gorman, M. Chemistry and Biology of $\beta$-Lactam Antibiotics; Acad. Press: New York, 1982.

7. Karchmer, A. W. In Mandell, Douglas and Bennet's Principle and Practice of Infectious Deseases; $5^{\text {th }}$ Edn.; Mandell, G. L.; Bennet, J. E.; Dolin, R., Eds.; Churchill Livingstone, Inc: Philadelphia, 2000, p 274.

8. Andriole, V. T. Drugs 1993, 45 Suppl.3, 1.

9. Hooper, D. C. In Mandell, Douglas and Bennet's Principle and Practice of Infectious Deseases; $5^{\text {th }}$ Edn.; Mandell, G. L.; Bennet, J. E.; Dolin, R.; Eds.; Churchill Livingstone, Inc: Philadelphia, 2000, p 404.

10. Pucci, M. J.; Hinks, E. T.; Dicker, D. T.; Higgins, M. L.; Daneo-Mur, L. J. Bacteriol. 1986, $165,682$.

11. Adu, A.; Armour, C. L. Drugs 1995, 50, 423.

12. Florey, K. Anal. Profiles Drug Subst. 1988, 17, 1.

13. Chantot, J. F.; Klich, M.; Teutsch, G.; Bryskier, A.; Collette, P.; Markus, A.; Seibert, G. Antimicrob. Agents Chemother 1992, 36, 1756.

14. Andrus, A.; Parttrige, B.; Heck, J. V.; Christensen, J. G. Tetrahedron Lett. 1984, $25,911$.

15. Brown, R. F.; Kinnick, M. D.; Morin, J. M.; Vasileff, R. T.; Counter, F. T.; Davidson, E. O.; Ensminger, P. W.; Eudaly, J. A.; Kasher, J. S.; Katner, A. S.; Koehler, R. E.; Kurz, K. D.; Lindstrom, T. D.; Lunn, W. H. W.; Preston, D. A.; Ott, J. L.; Quay, J. F.; Shadle, J. K.; Steinberg, M. I.; Stucky, J. F.; Swartzendruber, J. K.; Turner, J. R.; Webber, J. A.; Wright, W. E.; Zimmerman, K. M. J. Med. Chem. 1990, 33, 214.

16. Heider, J. Liebigs Ann. 1958, 613, 153.

17. Domagala, J. M. J. Org Chem. 1981, 46, 134.

18. Bradshaw, J.; Webber, G. B.; US Patent 3903 113, 1975.

19. Bonjouklian, R.; Phillips, M. L. Tetrahedron Lett. 1981, 22, 3915. 